\title{
Helicobacter pylori promotes VEGF expression via the p38 MAPK-mediated COX-2-PGE 2 pathway in MKN45 cells
}

\author{
NINGNING LIU ${ }^{1,2^{*}}$, QIONG WU ${ }^{2 *}$, YAN WANG $^{3}$, HUA SUI $^{2}$, XUAN LIU $^{2}$, NING ZHOU $^{2}$, LIHONG ZHOU $^{1}$, \\ YIFEI WANG ${ }^{1}$, NAIJING YE ${ }^{1}$, XIAOLING FU ${ }^{1}$, NIKITIN ALEXANDER YU ${ }^{4}$ and QI LI ${ }^{1,3}$ \\ ${ }^{1}$ Department of Medical Oncology, Shuguang Hospital; ${ }^{2}$ Department of Medical Oncology, Putuo Hospital; \\ ${ }^{3}$ Interventional Cancer Institute of Integrative Medicine and Putuo Hospital, Shanghai University of \\ Traditional Chinese Medicine, Shanghai 200062, P.R. China; ${ }^{4}$ Department of Biomedical Sciences, \\ Cornell University, Ithaca, New York 14853-6401, USA
}

Received July 19, 2013; Accepted March 12, 2014

DOI: $10.3892 / \mathrm{mmr} .2014 .2458$

\begin{abstract}
Helicobacter pylori has been suggested to be the major cause of gastric malignancy. However, the pathogenesis and molecular mechanisms of gastric tumorigenesis induced by $H$. pylori infection are yet to be elucidated. In the present study, the expression levels of vascular endothelial growth factor (VEGF), which has been suggested to promote angiogenesis in gastric cancer, were found to be elevated in $H$. pylori-infected MKN45 cells. Furthermore, it was demonstrated that the expression of VEGF was modulated by the p38 mitogen-activated protein kinases (MAPK) pathway via regulation of the cyclooxygenase (COX)-2 pathway. It was also found that prostaglandin $\mathrm{E} 2\left(\mathrm{PGE}_{2}\right)$ and its receptor EP2/EP4 may mediate the upregulation of VEGF in gastric cells exposed to H.pylori. In combination, these results suggest that VEGF expression is regulated by the $\mathrm{p} 38 \mathrm{MAPK} \mathrm{COX}-2-\mathrm{PGE}_{2}-\mathrm{EP} 2 / \mathrm{EP} 4$ pathway in gastric cancer cells induced by $H$. pylori. This provides a theoretical basis for the investigation of the pathogenesis of H. pylori-induced gastric cancer.
\end{abstract}

\section{Introduction}

Helicobacter pylori, a Gram-negative, spiral-shaped, microaerophilic bacterium found in the human stomach, is recognized as a major risk factor for chronic gastritis, peptic ulcers and gastric cancer (1). H. pylori infects approximately half of the world's population and has been classified as a carcinogen by the World Health Organization and the International Agency for Research on Cancer (2,3). Gastric cancer is the second

Correspondence to: Professor Qi Li, Department of Medical Oncology, Shuguang Hospital, Shanghai University of Traditional Chinese Medicine, 528 Zhangheng Road, Shanghai 201203, P.R. China

E-mail: lzwf@hotmail.com

Key words: Helicobacter pylori, VEGF, p38 MAPK, COX-2, $\mathrm{PGE}_{2}$, gastric cancer leading cause of cancer-associated mortality worldwide (4). Although considerable research has shown that $H$. pylori infection is closely associated with gastric cancer, the molecular mechanisms of gastric tumor initiation and development induced by $H$. pylori infection remain poorly understood (5).

Cyclooxygenase (COX), also known as prostaglandin-endoperoxide synthase, is a key rate-limiting enzyme responsible for the formation of prostanoids and thromboxanes $(6,7)$. There are three COX isoenzymes, COX-1, COX-2 and COX-3 (a splice variant of COX-1). COX-1 is considered a constitutive enzyme that is expressed in nearly all mammalian cells, whilst COX-2 is an inducible enzyme that is undetectable in the majority of normal tissues $(8,9)$. However, COX-2 expression is elevated in many types of cancer, including gastric cancer, and is closely correlated with the clinical outcome (10-15). Furthermore, a previous study demonstrated that COX-2 expression is associated with $H$. pylori infection in human gastric cancer, and the elevation of COX-2 expression may be mediated by the p38 mitogen-activated protein kinases (MAPK) pathway (16).

Prostaglandin E2 $\left(\mathrm{PGE}_{2}\right)$ is the main product generated from arachidonic acid catalyzed by $\mathrm{COX}-2$, and it exerts a wide range of pathological effects via receptors on the cell and nuclear membranes (17-19). Enhanced expression of $\mathrm{PGE}_{2}$ is found in several types of cancer and is associated with tumor growth and angiogenesis $(20,21)$. $\mathrm{PGE}_{2}$ receptors (EPs) are types of $\mathrm{G}$ protein-coupled receptor (GPCR), and exist in at least four isoforms: EP1, EP2, EP3 and EP4 (22-25). PGE binds to EPs to promote the expression of vascular endothelial growth factor (VEGF) in human prostate PC3 and gastric MKN28 cancer cells $(20,26)$. However, whether EPs mediate VEGF expression in gastric cancer cells following $H$. pylori infection, and the type of EP involved in such regulation, have yet to be elucidated.

The MAPK family of proteins are involved in cell differentiation, migration, apoptosis and autophagy (27). p38 MAPK is a key member of this family and it is part of a signaling cascade that modulates cellular responses to cytokines and stresses, including inflammatory cytokines, osmotic shock, lipopolysaccharide, ultraviolet light and growth factors (26). Previous studies have demonstrated that p38 MAPK is also activated following $H$. pylori infection, and promotes COX-2 
expression in MKN45 gastric cancer cells, which has been found to be involved in mediating $H$. pylori-induced gastric tumorigenesis (16,28-30). Several studies have investigated the oncogenic potential of the p38 MAPK pathway, since p38 MAPK also has a critical role in regulating VEGF expression leading to angiogenesis (27,31). However, whether p38 MAPK is involved in regulating VEGF expression in $H$.pylori-infected gastric cells is yet to be elucidated.

To investigate the mechanism of $H$. pylori-induced gastric cancer, VEGF expression was analyzed in MKN45 gastric cells following $H$. pylori infection. It was found that p38 MAPK has a critical role in regulating VEGF expression in $H$. pylori-infected MKN45 cells, and this effect may be mediated via the COX-2-EP2/EP4 pathway.

\section{Materials and methods}

Cell culture and reagents. MKN45 cells were obtained from the American Type Culture Collection (Manassas, VA, USA) and cultured in RPMI-1640 (Invitrogen ${ }^{\mathrm{TM}}$ Life Technologies, Carlsbad, CA, USA) containing 10\% (v/v) bovine serum albumin (BSA; Invitrogen Life Technologies) supplemented with $100 \mathrm{U} / \mathrm{ml}$ penicillin and $100 \mu \mathrm{g} / \mathrm{ml}$ streptomycin (Invitrogen Life Technologies). Cells were cultured in a humidified incubator containing $5 \% \mathrm{CO}_{2}$ at $37^{\circ} \mathrm{C}$. The p38 MAPK inhibitor SB203580, the COX-2 inhibitor $\mathrm{N}$-[2-(cyclohexyloxy)-4-nitrophenyl] methanesulfonamide (NS-398), the EP2 inhibitor AH6089 and the EP4 inhibitor AH23848 were all obtained from Cell Signaling Technology, Inc. (Danvers, MA, USA). For the inhibition treatment, confluent cells were treated with $20 \mu \mathrm{M}$ SB203580, $50 \mu \mathrm{M}$ NS-398, $50 \mu \mathrm{M}$ AH6089 or $50 \mu \mathrm{M}$ AH23848 for the indicated times.

H. pylori culture. The cagA- and vacA-positive $H$. pylori strain (NCTC11637) was acquired and cultured as previously described (15). In brief, $H$. pylori were cultured on Columbia Agar plates (Oxoid, Thermo Fisher Scientific, Basingstoke, UK) containing 10\% sheep blood and incubated at $37^{\circ} \mathrm{C}$ for $48-72 \mathrm{~h}$, with $5 \% \mathrm{O}_{2}, 10 \% \mathrm{CO}_{2}$ and $85 \% \mathrm{~N}_{2}$. Prior to use, H. pylori were identified using Gram staining, colony morphology and positive oxidase, catalase and urease reactions. To prepare $H$. pylori for infection, the cells were suspended in phosphate-buffered saline (PBS) and cell density was determined using spectrophotometry (Eppendorf BioSpectrometer; Eppendorf, Hamburg, Germany). Confluent MKN45 cells were then incubated with $H$. pylori at a quantity of 100 bacteria per cell for the indicated times.

RNA isolation and quantitative polymerase chain reaction $(q P C R)$. Total RNA was isolated from MKN45 cells using RNAisol Reagent kit (Takara Bio, Inc., Shiga, Japan) in accordance with the manufacturer's instructions. RNA quality was verified using spectrophotometry at an absorbance ratio of A260/280. Total RNA (1 $\mu \mathrm{g})$ was used for reverse transcription into cDNA using Prime-Script ${ }^{\mathrm{TM}}$ RT-PCR kit (Takara Bio, Inc.) under the following conditions: $37^{\circ} \mathrm{C}$ for $15 \mathrm{~min}$ and $85^{\circ} \mathrm{C}$ for $5 \mathrm{sec}$. A total of $1 \mu \mathrm{l} \mathrm{cDNA}$ was then used for qPCR amplification using the ABI 7300 Real-Time PCR system (Applied Biosystems, Foster City, CA, USA) under the following conditions: $95^{\circ} \mathrm{C}$ for $10 \mathrm{sec}, 95^{\circ} \mathrm{C}$ for $5 \mathrm{sec}$, and $60^{\circ} \mathrm{C}$ for $31 \mathrm{sec}$, run for 40 cycles. The forward and reverse primers for VEGF, COX-2 and GAPDH were used at a final concentration of $200 \mathrm{nM}$ and the sequences were as follows: VEGF, 5'-GGCCTCCGAAACCATGAACT-3' (forward) and 5'-CACTTGGCATGGTGGAGGTA-3' (reverse); COX-2, 5'-AATGAGTACCGCAAACGCTTCT-3' (forward) and 5'-TTCTGCAGCCATTTCCTTCTC-3' (reverse); GAPDH, 5'-CCACTCCTCCACCTTTGAC-3' (forward) and 5'-ACCCTGTTGCTGTAGCCA-3' (reverse). The TaqMan ${ }^{\circledR}$ probes (Invitrogen Life Technologies) used were as follows: 5'-TGTCTTGGGTGCATTGGAGC-3' for VEGF, 5'-CCTGAAGCCGTACACATCATTTG-3' for COX-2 and 5'-TTGCCCTCAACGACCACTTTGTC-3' for GAPDH.

Lenti-virus RNA interference (RNAi) plasmid construction and virus infection. The plasmids of lenti-virus RNAi system were obtained from Shanghai Genechem Co., Ltd. (Shanghai, China). Four small interfering RNAs (siRNAs) against human COX-2 mRNA [National Center for Biotechnology Information (NCBI) GenBank, NM-000963.2] were designed using the siRNA Target Finder from GenScript (Piscataway, NJ, USA). The target sequences used were as follows: Clone 1, 5'-GCT GAATTTAACACCCTCTAT-3' (1230-1250 bp); clone 2, 5'-CCATTCTCCTTGAAAGGACTT-3' (1677-1697bp); clone 3, 5'-GCAGATGAAATACCAGTCT T T-3' (1463-1483bp); and clone4,5'-CATTCCCTTCCTTCGAAAT-3' (407-425 bp). The clones were then inserted into a green fluorescent protein (GFP)-expressing pFU-GW-RNAi vector in accordance with the manufacturer's instructions. The pFU-GW-RNAi vector was co-transfected with pHelper 1.0 and pHelper 2.0 vectors into $293 \mathrm{~T}$ cells using Lipofectamine ${ }^{\circledR} 2000$ Transfection Reagent (Invitrogen Life Technologies). The virus was subsequently analyzed and amplified in 293T cells. The appropriate amount of virus was then used to infect MKN45 cells for $72 \mathrm{~h}$ in order to suppress the endogenous COX-2 expression.

Western blot analysis. Cells were lysed using lysis buffer solution (50 mM Tris- $\mathrm{HCl}, \mathrm{pH} 7.5 ; 150 \mathrm{mM} \mathrm{NaCl})$ containing $1 \%$ nonyl phenoxypolyethoxylethanol-40, $0.5 \%$ sodium deoxycholate, $0.1 \%$ SDS, $1 \mathrm{mM}$ phenylmethanesulfonylfluoride, $10 \mathrm{nM}$ microcystin, $1 \mu \mathrm{g} / \mathrm{ml}$ aprotinin and $1 \mu \mathrm{g} / \mathrm{ml}$ leupeptin (all constituents of the lysis buffer were purchased from Sangon Biotech Shanghai Co., Ltd., Shanghai, China). Following centrifugation at $14,000 \times \mathrm{g}$ for $20 \mathrm{~min}$, the protein in the supernatant was quantified using the BCA Protein Assay Reagent (Merck Millipore, Billerica, MA, USA) and equal amounts of protein were separated by $10 \%$ SDS-PAGE (Beyotime Institute of Biotechnology, Shanghai, China), prior to being transferred onto a polyvinylidene fluoride membrane (Bio-Rad, Hercules, CA, USA). Following blocking with 5\% fat-free milk in Tris-buffered saline in $0.05 \%$ Tween 20 (TBST; Sangon Biotech Shanghai Co., Ltd.) for $1 \mathrm{~h}$ at room temperature, the membranes were then separately incubated overnight at $4^{\circ} \mathrm{C}$ with the following antibodies: Rabbit anti-human COX-2 monoclonal antibody and rabbit anti-human $\beta$-actin monoclonal antibody (1:1,000; Cell Signaling Technology, Inc., Beverly, MA, USA), rabbit anti-human EP-2 polyclonal antibody and rabbit anti-human EP-4 polyclonal antibody 
(1:1,000; Abcam, Cambridge, UK). Membranes were rinsed three times with TBST (5 min each time) and then incubated with horseradish peroxidase-conjugated secondary antibodies (Jackson ImmunoResearch, West Grove, PA, USA) for $1 \mathrm{~h}$ at room temperature prior to visualization using the Pierce enhanced chemiluminescence kit (Pierce Biotechnology, Inc., Rockford, IL, USA). Results were analyzed using ImageJ software (National Institutes of Health, Bethesda, MD, USA).

ELISA. The cell culture medium was centrifuged at 3,000 x g for $5 \mathrm{~min}$, and the supernatant was then used for further analysis. ELISA was performed in accordance with the manufacturer's instructions (Invitrogen ${ }^{\mathrm{TM}}$ Life Technologies). Briefly, the microtiter plates were incubated with $100-\mu 1$ samples at $37^{\circ} \mathrm{C}$ for $120 \mathrm{~min}$. The plates were washed five times with $10 \mathrm{mM}$ PBS, prior to incubation with $100 \mu \mathrm{l}$ VEGF and $\mathrm{PGE}_{2}$ primary antibodies labeled with biotin at $37^{\circ} \mathrm{C}$ for $60 \mathrm{~min}$. The plates were then rinsed five times with $10 \mathrm{mM}$ PBS and $100 \mu 1$ avidin-biotin-peroxidase complex was added, prior to the plates being cultured at $37^{\circ} \mathrm{C}$ for $30 \mathrm{~min}$. Following extensive rinsing, the plates were then filled with $100 \mu 1 \mathrm{TMB}$ Microwell substrate and incubated in darkness at $37^{\circ} \mathrm{C}$ for $15 \mathrm{~min}$. The reaction was terminated using $100 \mu 1 \mathrm{TMB}$ stop solution and the optical density (OD) values were analyzed within 30 min using a microplate reader (Multiskan Spectrum; Thermo Fisher Scientific, Waltham, MA, USA) at $450 \mathrm{~nm}$. The OD values were subsequently converted into concentrations deduced from a calibration curve.

Statistical analysis. Statistical analysis was performed using the SPSS 19.0 software package (SPSS, Inc., Chicago, IL, USA). Statistical significance was determined using a one-way analysis of variance (ANOVA) followed by a Newman-Keuls test. All results are presented as the mean \pm standard error (SE) for three independent experiments. $\mathrm{P}<0.05$ was considered to indicate a statistically significant difference.

\section{Results}

H. pylori infection enhances expression levels of VEGF in MKN45 cells. To determine whether H. pylori induced VEGF expression, confluent MKN45 cells were co-cultured with $H$.pylori for $0,6,12$ and $24 \mathrm{~h}$. Cells were then harvested to examine the relative expression levels of VEGF mRNA compared with GAPDH mRNA expression levels using qPCR. It was found that VEGF mRNA levels in cells treated with H. pylori for $6 \mathrm{~h}(\mathrm{P}<0.05), 12 \mathrm{~h}(\mathrm{P}<0.01)$ and $24 \mathrm{~h}$ $(\mathrm{P}<0.01)$ were significantly elevated compared with cells not exposed to H.pylori (Fig. 1A). The VEGF mRNA expression was highest in cells treated with $H$. pylori for $12 \mathrm{~h}$. VEGF mRNA expression in cells treated with $H$. pylori for $12 \mathrm{~h}$ was significantly higher than that in cells treated for $6 \mathrm{~h}(\mathrm{P}<0.01)$; however, expression appeared to decline after $24 \mathrm{~h}$ (Fig. 1A). The VEGF protein expression levels were analyzed using ELISA. MKN45 cells were incubated with $H$. pylori for 0 , 12, 24, 36 and $48 \mathrm{~h}$, and the supernatant was harvested and analyzed. It was found that VEGF protein levels increased with the length of incubation, and were significantly elevated in cells cultured with $H$. pylori for $36 \mathrm{~h}(\mathrm{P}<0.05)$ and $48 \mathrm{~h}$ $(\mathrm{P}<0.01)$ (Fig. 1B).

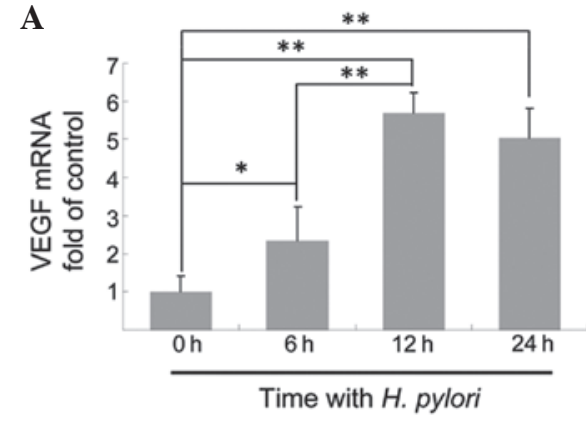

B

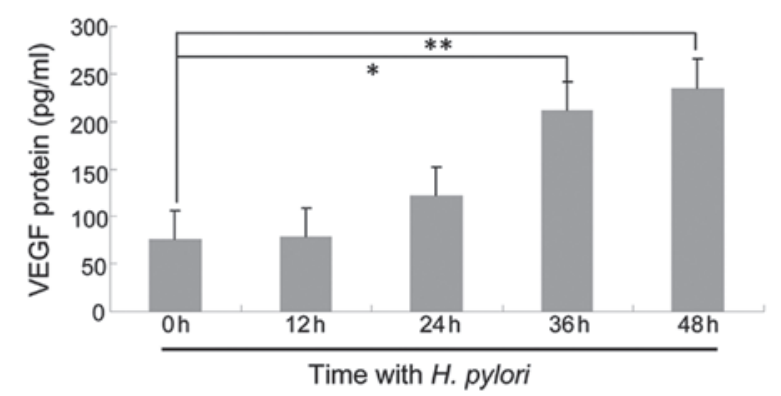

Figure 1. Time-dependent induction of VEGF expression by $H$. pylori in MKN45 cells. (A) Increase in VEGF mRNA expression in $H$. pylori-treated cells. Confluent MKN45 cells were incubated with $H$. pylori at a quantity of 100 bacteria per cell for $0,6,12$ and $24 \mathrm{~h}$. Cells were then harvested and analyzed using quantitative polymerase chain reaction to measure the relative expression levels of VEGF mRNA compared with GAPDH mRNA. (B) Increase in VEGF protein expression in $H$. pylori-treated cells. MKN45 cells were incubated with $H$. pylori for $0,12,24,36$ and $48 \mathrm{~h}$ and then harvested prior to analysis using ELISA to determine the VEGF protein expression levels. ${ }^{*} \mathrm{P}<0.05$ and ${ }^{* *} \mathrm{P}<0.01$. VEGF, vascular endothelial growth factor.

Inhibition of 38 MAPK attenuates the effects of $H$.pylori on VEGF expression. To examine whether $\mathrm{p} 38 \mathrm{MAPK}$ modulated VEGF expression, confluent MKN45 cells were pretreated with p38 MAPK inhibitor SB203580 for $2 \mathrm{~h}$ prior to incubation with or without $H$. pylori for 12 or $48 \mathrm{~h}$. Cells were then harvested and the levels of VEGF mRNA expression relative to GAPDH mRNA expression were analyzed using qPCR (cells treated with $H$. pylori for $12 \mathrm{~h}$ ), while VEGF protein expression levels were analyzed using ELISA (cells treated with $H$. pylori for $48 \mathrm{~h}$ ). In cells that were not exposed to H. pylori, treatment with SB203580 did not affect the VEGF mRNA or protein expression levels (Fig. 2A and B). Without SB203580 pretreatment, VEGF mRNA and protein levels in $H$. pylori-treated cells were significantly increased compared with cells not treated with H.pylori $(\mathrm{P}<0.01)$ (Fig. 2A and $\mathrm{B})$. However, when pretreated with SB203580, VEGF mRNA and protein expression levels in $H$. pylori-treated cells significantly decreased compared with $H$. pylori-treated cells without incubation with SB203580 (P<0.01) (Fig. 2A and B).

Blocking COX-2 with the inhibitor NS-398 attenuates the effects of $H$. pylori on VEGF expression. It was demonstrated in our previous study that p38 MAPK activity was essential for increased expression of COX-2 in MKN45 cells following $H$. pylori infection (15); therefore, in the present study, it was investigated whether COX-2 was involved in the p38 MAPK-mediated upregulation of VEGF 
A

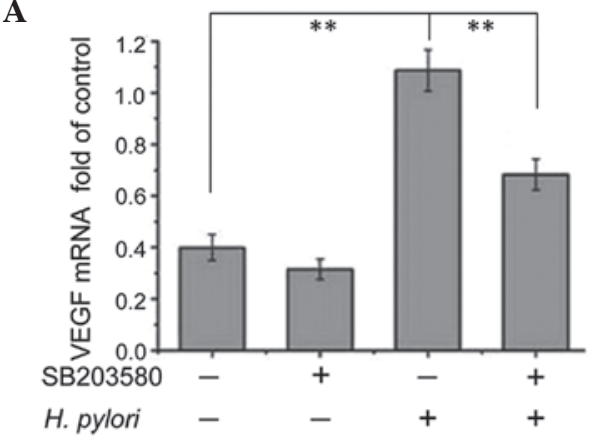

B

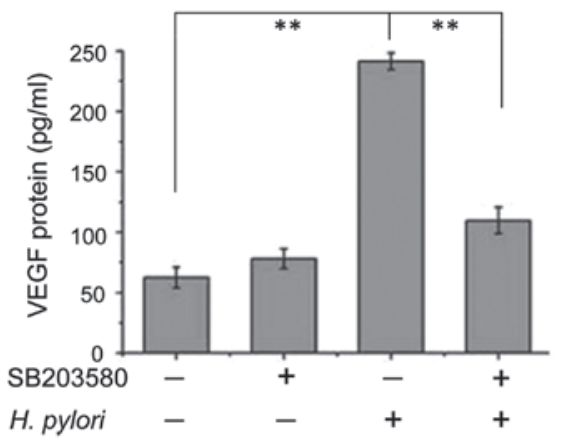

Figure 2. Effect of p38 MAPK inhibitor on VEGF expression following H. pylori infection in MKN45 cells. (A) p38 MAPK inhibitor SB203580 attenuates the effects of $H$. pylori on VEGF mRNA expression. Confluent MKN45 cells were pretreated with $20 \mu \mathrm{M}$ p38 MAPK inhibitor SB203580 for $2 \mathrm{~h}$ prior to co-culture with or without $H$. pylori for $12 \mathrm{~h}$. The cells were then harvested to measure the relative expression levels of VEGF mRNA compared with GAPDH mRNA using quantitative polymerase chain reaction. (B) p38 MAPK inhibitor SB203580 attenuates the effects of $H$. pylori on VEGF protein expression. Confluent MKN45 cells were pretreated with $20 \mu \mathrm{M}$ p38 MAPK inhibitor SB203580 for $2 \mathrm{~h}$ prior to co-culture with or without $H$. pylori for $12 \mathrm{~h}$. The cells were then harvested to measure the relative protein expression levels of VEGF using ELISA. ${ }^{* *} \mathrm{P}<0.01$. p38 MAPK, p38 mitogen-activated protein kinase; VEGF, vascular endothelial growth factor.

expression. Confluent MKN45 cells were pretreated with the COX-2 inhibitor NS-398 for $2 \mathrm{~h}$ prior to incubation with or without $H$. pylori for $12 \mathrm{~h}$. The cells were then harvested to measure the expression levels of VEGF mRNA compared with GAPDH mRNA using qPCR. In cells not exposed to H. pylori, treatment with NS-398 did not affect the VEGF mRNA expression levels (Fig. 3A). Consistent with our previous results, VEGF mRNA levels in $H$. pylori-treated cells increased significantly $(\mathrm{P}<0.01)$ (Fig. 3A). However, in $H$. pylori-infected cells pretreated with NS-398, VEGF mRNA expression levels were downregulated compared with H. pylori-infected cells not incubated with NS-398 $(\mathrm{P}<0.01)$ (Fig. 3A). Our previous studies demonstrated that $H$. pylori increased COX-2 expression in MKN45 cells (16); therefore, it was hypothesized that the downstream products of COX-2 may have been elevated. The present study analyzed the protein expression levels of $\mathrm{PGE}_{2}$, one such downstream product of COX-2, in MKN45 cells incubated with $H$. pylori for $0,6,12,24$ and $48 \mathrm{~h}$ using ELISA. PGE $_{2}$ protein levels in cells treated with $H$. pylori for 12,24 and $48 \mathrm{~h}$ were significantly higher compared with cells not exposed to $H$. pylori $(\mathrm{P}<0.01)$, with the highest expression observed in in cells treated with H.pylori for $24 \mathrm{~h}$ (Fig. 3B).

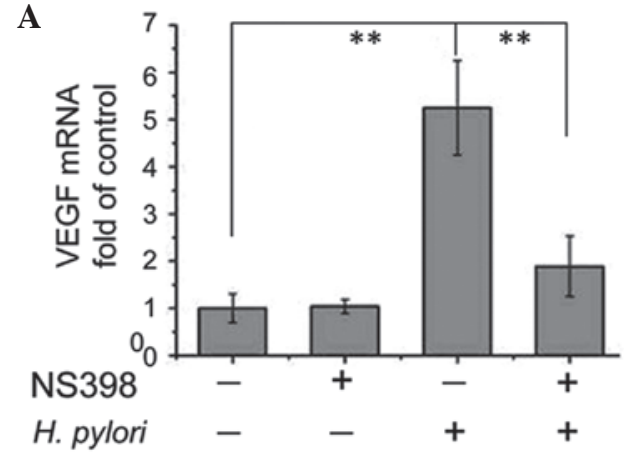

\section{B}

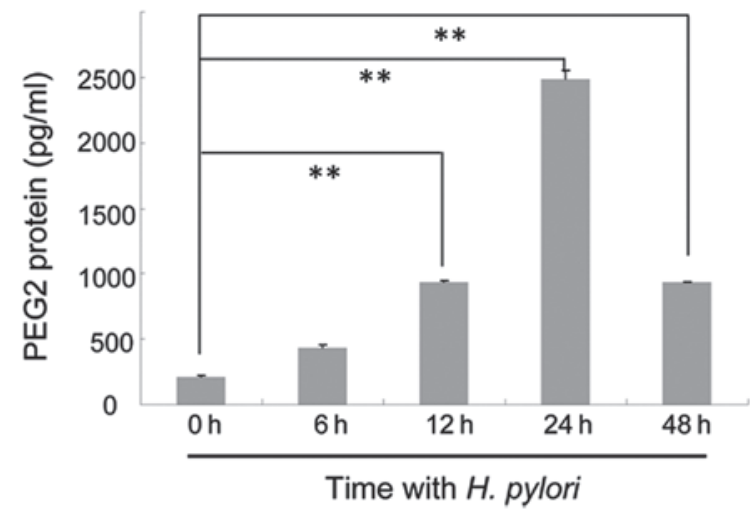

Figure 3. Effect of COX-2 inhibitor on VEGF expression following H.pylori infection in MKN45 cells. (A) COX-2 inhibitor NS-398 attenuates the effects of $H$. pylori on VEGF mRNA expression. Confluent MKN45 cells were pretreated with $50 \mu \mathrm{M} \mathrm{COX}-2$ inhibitor NS-398 for $2 \mathrm{~h}$ prior to co-culture with or without $H$. pylori for $12 \mathrm{~h}$. Cells were then harvested and the relative levels of VEGF mRNA expression compared with GAPDH mRNA were analyzed using quantitative polymerase chain reaction. (B) Increase in $\mathrm{PGE}_{2}$ protein expression in $H$. pylori-treated cells. MKN45 cells were incubated with $H$. pylori for $0,6,12,24$ and $48 \mathrm{~h}$ and supernatant was harvested for ELISA analysis to measure the protein expression of $\mathrm{PGE}_{2}$, one downstream product of COX-2. ${ }^{* *} \mathrm{P}<0.01$. VEGF, vascular endothelial growth factor; COX-2, cyclooxygenase-2; NS-398, N-[2-(cyclohexyloxy)-4-nitrophenyl] methanesulfonamide.

RNAi-mediated suppression of COX-2 attenuates the effects of H. pylori on VEGF expression. In order to specifically suppress endogenous COX-2 expression, four lentiviral based RNAi clones targeted to different parts of the COX-2 gene were designed, and the inhibitory effect was analyzed by infecting MKN45 cells for $72 \mathrm{~h}$ with each clone separately. The cells were then harvested and the expression levels of COX-2 mRNA relative to GAPDH mRNA expression were analyzed using qPCR, while COX-2 protein expression was measured using western blot analysis. All four of the clones of lenti-viral RNAi targeted to COX-2 efficiently suppressed mRNA and protein expression levels of COX-2; however, clone 4 suppressed COX-2 mRNA and protein expression levels by $\sim 95$ and $\sim 81 \%$, respectively and, therefore, was used in the following experiments (Fig. 4A and B). To determine the effects of COX-2 RNAi on VEGF expression, confluent MKN45 cells were infected with control RNAi (GFP control), or COX-2 RNAi for $72 \mathrm{~h}$, and then cells were incubated with or without $H$. pylori for $48 \mathrm{~h}$. The supernatant was harvested to measure the protein expression of VEGF using ELISA. The results showed that background expression of VEGF in 
A

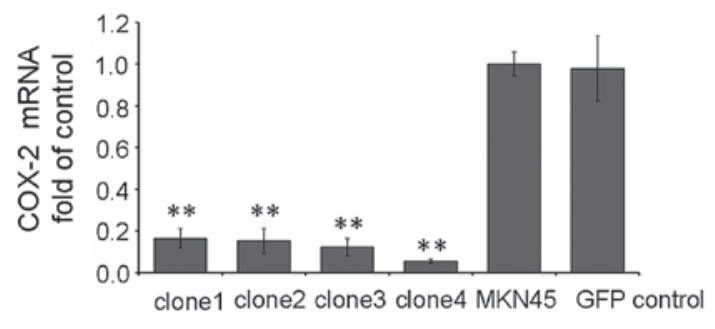

B

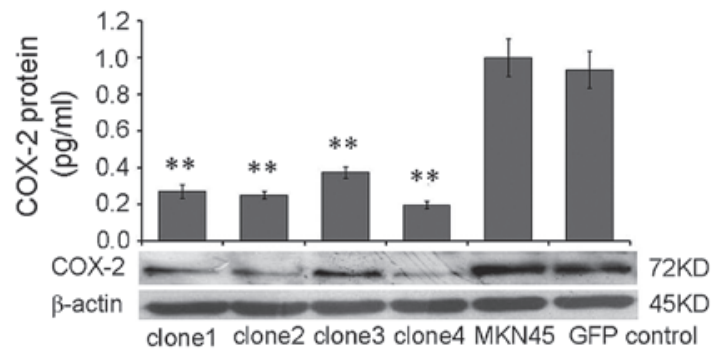

C

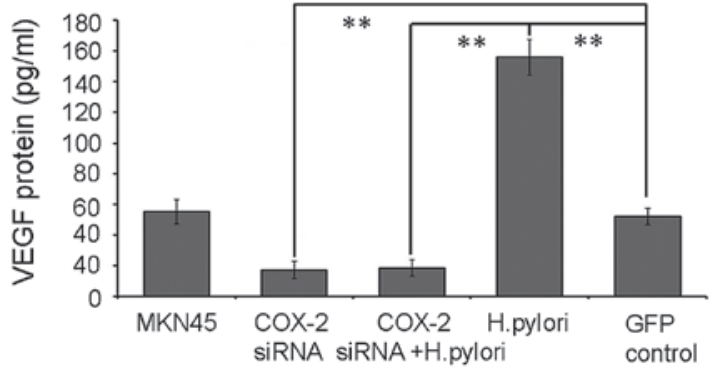

Figure 4. Effect of COX-2 RNAi on VEGF expression following H. pylori infection in MKN45 cells. (A and B) COX-2 RNAi largely suppresses endogenous COX-2 (A) mRNA and (B) protein expression levels. Confluent MKN45 cells were infected with control lenti-viral RNAi (GFP) or four clones of lenti-viral RNAi of COX-2 for $72 \mathrm{~h}$. Cells were then harvested to measure the relative expression levels of COX-2 mRNA and protein using quantitative polymerase chain reaction and western blot analysis, respectively. ${ }^{* *} \mathrm{P}<0.01$, compared with the GFP control group. (C) RNAi-mediated inhibition of COX-2 aborts the upregulation of VEGF expression following H. pylori infection. Confluent MKN45 cells were infected with control lenti-viral RNAi (GFP) or clone 4 of the lenti-viral RNAi of COX-2 for $72 \mathrm{~h}$ and incubated with or without $H$. pylori for $48 \mathrm{~h}$. The supernatant was harvested and the protein expression of VEGF was analyzed using ELISA ${ }^{* *} \mathrm{P}<0.01$. COX-2, cyclooxygenase-2; RNAi, RNA interference; VEGF, vascular endothelial growth factor; GFP, green fluorescent protein.

MKN45 cells was significantly reduced in cells infected with COX-2 siRNA $(\mathrm{P}<0.01)$, and the same effect was observed in cells also treated with $H$. pylori. This indicates that RNAi-mediated suppression of COX-2 inhibits the upregulation of VEGF expression in MKN45 cells following H.pylori treatment (Fig. 4C).

EP2/EP4-mediated regulation of VEGF expression upon $H$. pylori infection. Since it was found that COX-2 promotes VEGF expression in $H$. pylori-treated MKN45 cells and that the downstream product of $\mathrm{COX}-2, \mathrm{PGE}_{2}$, was upregulated, further studies were performed to determine whether the $\mathrm{PGE}_{2}$ receptors EP2/EP4 were also associated with COX-2-mediated upregulation of VEGF. Confluent MKN45 cells were incubated with $H$. pylori for $0,2,6,12,24$ and $48 \mathrm{~h}$ and then harvested. Western blot analysis was performed to determine the protein expression levels of EP2 and EP4, using $\beta$-actin as the internal control. As shown in Fig. 5A,
A

Time with H. pylori

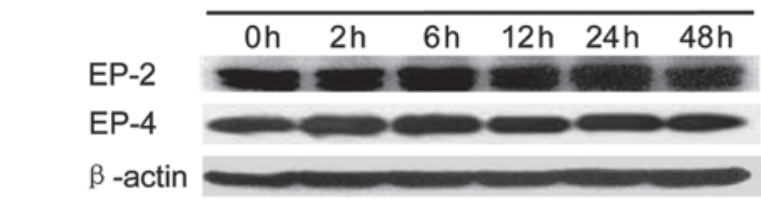

B

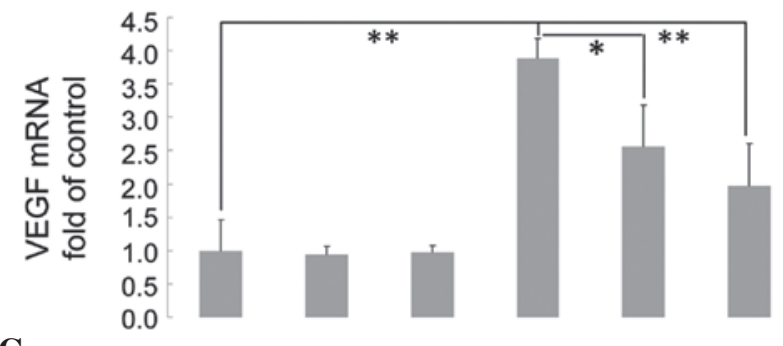

C

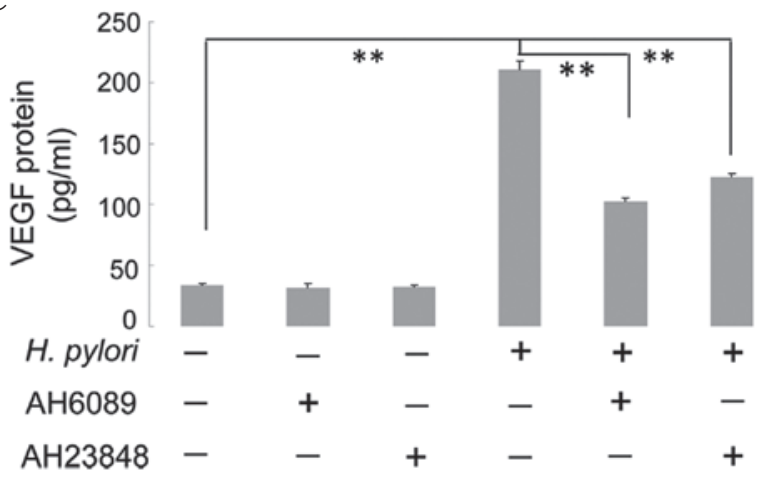

Figure 5. Effect of EP2/EP4 inhibitors on H. pylori-induced VEGF expression in MKN45 cells. (A) Protein expression of EP2 and EP4 does not change in $\mathrm{H}$. pylori-treated MKN45 cells. Confluent MKN45 cells were co-cultured with $H$. pylori for $0,2,6,12,24$ and $48 \mathrm{~h}$, prior to western blot analysis to measure the expression of EP2 and EP4. $\beta$-actin was used as an internal control. (B) The inhibitors AH6089 and AH23848 attenuate the effects of H. pylori on VEGF mRNA expression. Confluent cells were pretreated for $2 \mathrm{~h}$ with $50 \mu \mathrm{M}$ specific inhibitors against EP2 or EP4, AH6089 and AH23848, respectively. Cells were then incubated with or without $H$. pylori for $12 \mathrm{~h}$, and harvested to measure the relative expression levels of VEGF mRNA compared with GAPDH using quantitative polymerase chain reaction. (C) The inhibitors AH6089 and AH23848 attenuate the effects of $H$.pylori on VEGF protein expression. Confluent cells were pretreated for $2 \mathrm{~h}$ with $50 \mu \mathrm{M}$ specific inhibitors against EP2 or EP4, AH6089 and AH23848, respectively. Cells were then incubated with or without $H$. pylori for $48 \mathrm{~h}$, and harvested to measure the expression levels of VEGF protein using ELISA. " $\mathrm{P}<0.05$ and ${ }^{* *} \mathrm{P}<0.01$. EP, prostaglandin $\mathrm{E} 2$ receptor; VEGF, vascular endothelial growth factor.

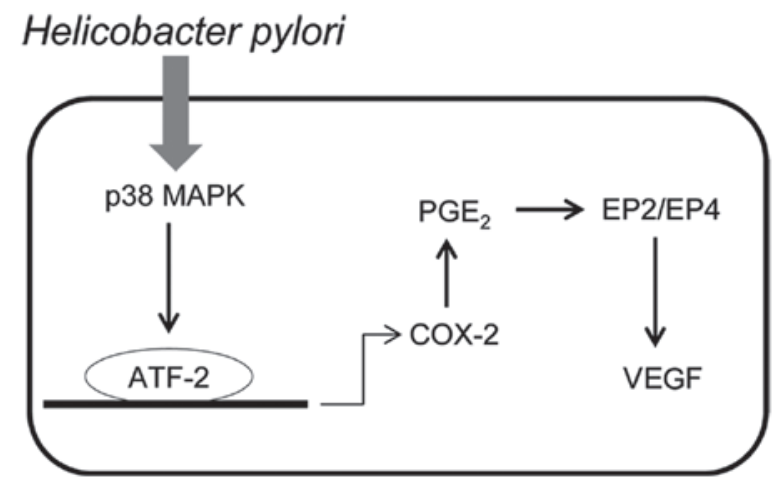

Figure 6. Model of the upregulation of VEGF expression via p38 MAPK-mediated COX-2-PGE 2 pathway following $H$. pylori infection in MKN45 cells. VEGF, vascular endothelial growth factor; p38 MAPK, p38 mitogen-activated protein kinase; ATF-2, activating transcription factor 2; $\mathrm{COX}-2$, cyclooxygenase-2; $\mathrm{PGE}_{2}$, prostaglandin $\mathrm{E} 2 ; \mathrm{EP}, \mathrm{PGE}_{2}$ receptor. 
no significant difference was observed in the protein expression levels of EP2 or EP4 during the continual induction of H. pylori (Fig. 5A). To examine whether EP2/EP4 expression was associated with VEGF expression in MKN45 cells, confluent cells were pretreated with specific inhibitors against EP2 and EP4, AH6089 and AH23848, respectively, for $2 \mathrm{~h}$ prior to incubation with or without H.pylori for 12 or $48 \mathrm{~h}$. The cells were then harvested and the expression levels of VEGF mRNA relative to GAPDH mRNA were analyzed using qPCR (cells treated with $H$. pylori for $12 \mathrm{~h}$ ), while expression levels of VEGF protein were analyzed using ELISA (cells treated with $H$. pylori for 48 h). In cells not exposed to H. pylori, treatment with AH6089 or AH23848 did not affect VEGF mRNA or protein expression levels ( $\mathrm{P}>0.05$ ) (Fig. 5B and $\mathrm{C}$ ). In cells not treated with AH0689 or AH2348, VEGF mRNA and protein levels in $H$. pylori-infected cells increased significantly compared with cells not infected with H.pylori $(\mathrm{P}<0.01)$ (Fig. 5B and C). However, when cells were pretreated with AH6089, VEGF mRNA $(\mathrm{P}<0.05)$ and protein $(\mathrm{P}<0.01)$ expression levels in $H$. pylori-infected cells significantly decreased compared with $H$. pylori-infected cells without incubation of AH6089 (Fig. 5B and C). A similar result was observed in cells pretreated with the inhibitor AH23848: VEGF mRNA and protein expression levels in $H$. pylori-infected cells significantly decreased compared with $H$. pylori-infected cells without incubation of AH23848 ( $<<0.01)$ (Fig. 5B and C).

\section{Discussion}

VEGF, an oncogenic marker in cancer diagnosis, has potent angiogenic activity on endothelial cells and promotes tumor growth (32). Enhanced expression of VEGF is often observed in malignant tumors and is frequently used as a therapeutic target (32). A previous study demonstrated that H. pylori infection promoted gastric cancer cell invasion via upregulation of VEGF expression (33). However, the mechanism by which $H$. pylori induces VEGF expression in gastric cancer has yet to be elucidated. The p38 MAPK pathway has been found to be involved in tumor growth and metastasis through regulation of the production of VEGF in cancer. p38 MAPK activity also has a key role in increasing $H$. pylori-induced COX-2 expression in gastric cancer cells $(16,27,31)$. Therefore, in the present study, the role of p38 MAPK in the regulation of VEGF expression in gastric cancer cells exposed to $H$. pylori was investigated. It was found that mRNA and protein expression levels of VEGF were significantly increased following H.pylori infection; however, the p38 MAPK-specific inhibitor, SB203580, significantly attenuated this effect, indicating that p38 MAPK was involved in promoting VEGF expression in H. pylori-infected MKN45 cells (Figs. 1 and 2).

COX-2 activity is associated with $H$. pylori infection in gastric cells, and may promote the production of $\mathrm{PGE}_{2}(16,26)$. In a previous study, we demonstrated that $H$. pylori infection upregulates the expression of COX-2 via the p38 MAPK/activating transcription factor 2 pathway (16). In this present study, the expression of $\mathrm{PGE}_{2}$ in $H$. pylori-infected cells was analyzed and it was found that $\mathrm{PGE}_{2}$ levels were significantly increased, suggesting that COX-2 activity was upregulated following $H$. pylori infection (Fig. 3B). To investigate whether the p38 MAPK-associated upregulation of VEGF expression was mediated by COX-2, the COX-2 specific inhibitor, NS-398, was used in cells exposed to $H$. pylori. The results show that VEGF expression significantly decreased in NS-398-treated cells (Fig. 3A). Endogenous COX-2 expression was also downregulated using RNAi and it was found that background expression levels of VEGF in MKN45 cells were significantly reduced, and the same effect was observed in $H$. pylori-treated cells, indicating that RNAi-mediated inhibition of COX-2 suppressed the upregulation of VEGF expression in MKN45 cells following H. pylori infection (Fig. 4C).

The results of the present study suggest that COX-2 is involved in the regulation of VEGF expression, downstream of p38 MAPK. EP2 and EP4 are $\mathrm{PGE}_{2}$ receptors, and have been shown to have an important role in modulating VEGF production in prostate cancer cells $(20,26)$. In the present study, the roles of EP2/EP4 in VEGF production in gastric cancer cells following $H$. pylori infection were investigated. The results demonstrated that inhibition of EP2 and EP4 with the specific inhibitors AH6089 and AH23848 significantly decreased $H$. pylori-induced VEGF levels in cells, indicating that EP2/EP4 mediate the upregulation of VEGF expression in $H$. pylori-infected gastric cancer cells (Fig. 5B and C). The protein expression of EP2/EP4 was then analyzed, and it was found that $H$. pylori infection did not alter the EP2/EP4 protein levels. This suggests that the EP2/EP4-associated upregulation of VEGF is not mediated by EP2/EP4 protein levels, but by increased levels of $\mathrm{PGE}_{2}$ product, induced by enhanced COX-2 activity (Fig. 5A).

In combination, these results suggest a novel pathway of p38 MAPK-COX-2-PGE 2 -EP2/EP4 for the regulation of VEGF expression in $H$. pylori-infected gastric cells (Fig. 6). Following $H$. pylori infection, p38 MAPK is activated and COX-2 expression levels are upregulated, the level of $\mathrm{PGE}_{2}$ is therefore increased. $\mathrm{PGE}_{2}$ then binds to $\mathrm{EP} 2 / \mathrm{EP} 4$ to promote VEGF expression. In a previous study we demonstrated that Jianpi Jiedu, a formulation used in traditional Chinese medicine, was demonstrated to downregulate COX-2 expression via inhibition of the H. pylori-induced p38 MAPK pathway (34). Therefore, further studies may be performed to examine whether the Jianpi Jiedu recipe regulates VEGF expression via modulation of this pathway, as shown in Fig. 6.

In conclusion, the present study elucidated a p38 MAPK-mediated signaling pathway that regulates VEGF expression in $H$. pylori-infected gastric cancer cells. This contributes to the investigation into the pathogenesis of H. pylori-induced gastric cancer. Furthermore, this study may provide novel therapeutic targets for $H$. pylori-induced gastric cancer.

\section{Acknowledgements}

This study was funded and supported by the National Natural Science Foundation of China (nos. 81072955, 81273958 and 81202663), the Program of Shanghai Municipal Education Commission (12ZZ118), the Science and Technology Commission of Shanghai Municipality (12ZR1449300, 1214090250), the Shanghai Municipal Health Bureau (2010019, XBR2011061, 2010044) and the Major Program of Technology Innovation, Putuo District, Shanghai (2009PTKW001). 


\section{References}

1. Peek RM Jr and Crabtree JE: Helicobacter infection and gastric neoplasia. J Pathol 208: 233-248, 2006.

2. Ernst PB, Peura DA and Crowe SE: The translation of Helicobacter pylori basic research to patient care. Gastroenterology 130: 188-206; quiz 212-213, 2006.

3 . No authors listed: IARC working group on the evaluation of carcinogenic risks to humans: some industrial chemicals. Lyon, 15-22 February 1994. IARC Monogr Eval Carcinog Risks Hum 60: 1-560, 1994.

4. Wroblewski LE, Peek RM, Jr and Wilson KT: Helicobacter pylori and gastric cancer: factors that modulate disease risk. Clin Microbiol Rev 23: 713-739, 2010.

5. Conteduca V, Sansonno D, Lauletta G, Russi S, Ingravallo G and Dammacco F: $H$. pylori infection and gastric cancer: state of the art (review). Int J Oncol 42: 5-18, 2013.

6. de Vries EF: Imaging of cyclooxygenase-2 (COX-2) expression: potential use in diagnosis and drug evaluation. Curr Pharm Des 12 3847-3856, 2006.

7. Greenhough A, Smartt HJ, Moore AE, et al: The COX-2/PGE2 pathway: key roles in the hallmarks of cancer and adaptation to the tumour microenvironment. Carcinogenesis 30: 377-386, 2009.

8. Zidar N, Dolenc-Strazar Z, Jeruc J, et al: Expression of cyclooxygenase-1 and cyclooxygenase-2 in the normal human heart and in myocardial infarction. Cardiovasc Pathol 16: 300-304, 2007.

9. Zidar N, Odar K, Glavac D, Jerse M, Zupanc T and Stajer D: Cyclooxygenase in normal human tissues - is COX-1 really a constitutive isoform, and COX-2 an inducible isoform? J Cell Mol Med 13: 3753-3763, 2009.

10. Buskens CJ, Van Rees BP, Sivula A, et al: Prognostic significance of elevated cyclooxygenase 2 expression in patients with adenocarcinoma of the esophagus. Gastroenterology 122: 1800-1807, 2002.

11. Erkinheimo TL, Lassus H, Sivula A, et al: Cytoplasmic HuR expression correlates with poor outcome and with cyclooxygenase 2 expression in serous ovarian carcinoma. Cancer Res 63: 7591-7594, 2003.

12. Juuti A, Louhimo J, Nordling S, Ristimäki A and Haglund C: Cyclooxygenase-2 expression correlates with poor prognosis in pancreatic cancer. J Clin Pathol 59: 382-386, 2006.

13. Menter DG, Schilsky RL and DuBois RN: Cyclooxygenase-2 and cancer treatment: understanding the risk should be worth the reward. Clin Cancer Res 16: 1384-1390, 2010.

14. Ristimäki A, Sivula A, Lundin J, et al: Prognostic significance of elevated cyclooxygenase- 2 expression in breast cancer. Cancer Res 62: 632-635, 2002.

15. Thiel A, Mrena J and Ristimäki A: Cyclooxygenase-2 and gastric cancer. Cancer Metastasis Rev 30: 387-395, 2011.

16. Li Q, Liu N, Shen B, et al: Helicobacter pylori enhances cyclooxygenase 2 expression via p38MAPK/ATF-2 signaling pathway in MKN45 cells. Cancer Lett 278: 97-103, 2009.

17. Fujino H, Xu W and Regan JW: Prostaglandin E2 induced functional expression of early growth response factor-1 by EP4, but not EP2, prostanoid receptors via the phosphatidylinositol 3-kinase and extracellular signal-regulated kinases. J Biol Chem 278: 12151-12156, 2003

18. Golijanin D, Tan JY, Kazior A, et al: Cyclooxygenase-2 and microsomal prostaglandin E synthase-1 are overexpressed in squamous cell carcinoma of the penis. Clin Cancer Res 10: 1024-1031, 2004.
19. Sheng H, Shao J, Washington $M K$ and DuBois RN: Prostaglandin E2 increases growth and motility of colorectal carcinoma cells. J Biol Chem 276: 18075-18081, 2001.

20. Ding YB, Shi RH, Tong JD, et al: PGE2 up-regulates vascular endothelial growth factor expression in MKN28 gastric cancer cells via epidermal growth factor receptor signaling system. Exp Oncol 27: 108-113, 2005.

21. Pai R, Szabo IL, Soreghan BA, Atay S, Kawanaka H and Tarnawski AS: PGE(2) stimulates VEGF expression in endothelial cells via ERK2/JNK1 signaling pathways. Biochem Biophys Res Commun 286: 923-928, 2001.

22. Araki H, Ukawa H, Sugawa Y, Yagi K, Suzuki K and Takeuchi K: The roles of prostaglandin E receptor subtypes in the cytoprotective action of prostaglandin E2 in rat stomach. Aliment Pharmacol Ther 14 (Suppl 1): 116-124, 2000.

23. Breyer RM, Emeson RB, Tarng JL, et al: Alternative splicing generates multiple isoforms of a rabbit prostaglandin E2 receptor. J Biol Chem 269: 6163-6169, 1994.

24. Pang L and Knox AJ: Bradykinin stimulates IL-8 production in cultured human airway smooth muscle cells: role of cyclooxygenase products. J Immunol 161: 2509-2515, 1998.

25. Takeuchi K, Araki H, Umeda M, Komoike Y and Suzuki K: Adaptive gastric cytoprotection is mediated by prostaglandin EP1 receptors: a study using rats and knockout mice. J Pharmacol Exp Ther 297: 1160-1165, 2001.

26. Jain S, Chakraborty G, Raja R, Kale S and Kundu GC: Prostaglandin E2 regulates tumor angiogenesis in prostate cancer. Cancer Res 68: 7750-7759, 2008.

27. Cuenda A and Rousseau S: p38 MAP-kinases pathway regulation, function and role in human diseases. Biochim Biophys Acta 1773: 1358-1375, 2007

28. Choi IJ, Kim JS, Kim JM, Jung HC and Song IS: Effect of inhibition of extracellular signal-regulated kinase 1 and 2 pathway on apoptosis and bcl-2 expression in Helicobacter pylori-infected AGS cells. Infection Immun 71: 830-837, 2003.

29. Kim H,Seo JH and Kim KH: The effect of p38 mitogen-activated protein kinase on mucin gene expression and apoptosis in Helicobacter pylori-infected gastric epithelial cells. Ann NY Acad Sci 1010: 90-94, 2003.

30. Seo JH, Lim JW, Kim H and Kim KH: Helicobacter pylori in a Korean isolate activates mitogen-activated protein kinases, AP-1, and NF-kappaB and induces chemokine expression in gastric epithelial AGS cells. Lab Invest 84: 49-62, 2004.

31. Kim JH, Studer RK, Vo NV, Sowa GA and Kang JD: p38 MAPK inhibition selectively mitigates inflammatory mediators and VEGF production in AF cells co-cultured with activated macrophage-like THP-1 cells. Osteoarthritis Cartilage 17: 1662-1669, 2009.

32. Goel HL and Mercurio AM: VEGF targets the tumour cell. Nat Rev Cancer 13: 871-882, 2013.

33. Wu CY, Wang CJ, Tseng CC, et al: Helicobacter pylori promote gastric cancer cells invasion through a NF-kappaB and COX-2-mediated pathway. World J Gastroenterol 11: 3197-3203, 2005.

34. Liu NN, Wang Y and Wu Q: Jianpi jiedu recipe inhibited Helicobacter pylori-induced the expression of cyclooxygenase-2 via p38MAPK/ATF-2 signal transduction pathway in human gastric cancer cells. Zhongguo Zhong Xi Yi Jie He Za Zhi 31: 926-931, 2011 (In Chinese). 